\title{
Genetic investigations on the caffeine and chlorogenic acid relationship in an interspecific cross between Coffea liberica dewevrei and $C$. pseudozanguebariae
}

\author{
Chin-Long $\mathrm{Ky}^{1}$, Philippe Barre ${ }^{2}$, Michel Noirot ${ }^{3, *}$ \\ ${ }^{1}$ Centre IFREMER de Tahiti, Taravao, French Polynesia \\ ${ }^{2}$ Centre INRA de Lusignan, Lusignan, France \\ ${ }^{3}$ IRD, UMR PVBMT, Centre CIRAD Pôle de Protection des Plantes, 97410 St Pierre, La Réunion, France \\ *: Corresponding author : Michel Noirot, email address : noirot@cirad.fr
}

\begin{abstract}
:
The objective of the paper was to identify the number of major loci explaining caffeine content in coffee seeds. Investigations were based on previously published results: (1) Caffeine binds to chlorogenic acids in a 1:1 molecular ratio; (2) Between species, the caffeine content is correlated to the chlorogenic acid content; (3) Only a part of chlorogenic acids is bound to caffeine. Especially, the content ratio between caffeine and chlorogenic acids varied between species. For identifying the number of major loci, a quantitative trait locus (QTL) approach was carried out using an interspecific cross between two highly differentiated species-Coffea liberica dewevrei and Coffea pseudozanguebariae, the latter being a caffeine-free species. As main finding, two QTLs, i.e., RCQ1 and CQA1, were identified allowing us to explain up to $97 \%$ of the caffeine content variance. RCQ1 explained variation of the caffeine/chlorogenic acid ratio and was genetically independent of the second QTL. The latter explained the part of the caffeine content which was dependent on the chlorogenic acid content. The findings also confirmed that only a part of chlorogenic acids were trapped by caffeine, as in wild species.
\end{abstract}

Keywords: Coffea ; Chlorogenic acids ; Caffeine ; QTL ; Interspecific cross 


\section{Introduction}

Caffeine is a secondary metabolite of great interest in coffee production because of its impact on beverage quality (its accumulation in green coffee beans increases bitterness of the beverage). It is mainly known to influence the central nervous system, especially with respect to insomnia (Nehlig et al. 1992). Nevertheless, recent studies also report the beneficial properties of moderate doses of caffeine concerning Parkinson"s or Alzheimer"s disease, type 2 diabetes and numerous cancers (Nehlig 2012). This explains current research aimed at identifying genes involved in its biosynthesis (Ogawa et al. 2001; Mizuno et al. 2003; Uefuji et al. 2003) or obtaining genetically modified varieties with lower caffeine content (CAF) (Ogita et al. 2003). In the present paper, the objective was to determine the number of major loci explaining CAF in coffee trees. For this, a QTL approach was carried out using an interspecific cross between two species with highly differentiated caffeine content. Present investigations were also based on the known relationships between CAF and chlorogenic acid content (CGA) in green beans.

In green beans of African coffee species, the CAF varies markedly between species, from $0 \%$ dry matter basis (dmb) in C. pseudozanguebariae (PSE) to $2.5 \%$ in C. canephora (Charrier and Berthaud 1975; Anthony et al. 1993; Mazzafera et al. 1997; Ky et al. 2001; Campa et al. 2005a). Nevertheless, caffeine-free species actually have a trace of caffeine $(<0.01 \% \mathrm{dmb})$ in their green beans (Campa et al. 2005a). Most of the variation (about $94 \%$ ) is under genetic control (Barre et al. 1998; Charrier and Berthaud 1975; Montagnon et al. 1998).

Chlorogenic acids sensu largo (CGA) comprise three major subgroups representing $98 \%$ of all CGAs (Clifford, 1985). These three groups are quinic acid esters with caffeic acid [caffeoylquinic acids (CQA) and dicaffeoylquinic acids (diCQA)] or with ferulic acid [feruloylquinic acids (FQA)]. Three isomers exist within each group and the most abundant is the 5-CQA, known as chlorogenic acid sensu stricto.

A complex between chlorogenic acids and caffeine is known since the $19^{\text {th }}$ century (Payen 1846) and occurs at a 1:1 molecular ratio (Sondheimer et al. 1961). Nevertheless, several species show similar chlorogenic acid content (CGA), but different CAF. This is the case in C. eugenioides and C. racemosa which have a CGA of $5.17 \%$ and $5.33 \%$ dmb, respectively, whereas CAF is $0.51 \%$ and $1.05 \%$ dmb, respectively (Campa et al. 2005b). C. pocsii and C. brevipes provide a second example. In these species, CGAs are close $(10.7 \%$ and $11.2 \%$ dmb, respectively), whereas CAF markedly differs $(1.27 \%$ and $2.54 \%$ dmb, respectively) (Campa et al. 2005b). This means that the CAF/CGA ratio varies between species and that only a part of these chlorogenic acids are complexed with caffeine. In C. eugenioides, $1 \%$ $\mathrm{dmb}$ of CGA is present as caffeine chlorogenate and $4.4 \% \mathrm{dmb}(81.5 \%$ of total CGA) as caffeine-free CGA. This suggests genetic regulation of the complexation and, consequently, a possible effect of this regulation on the caffeine content.

The interspecific cross allowing here investigations on genetic factors influencing CAF has for parents the caffeine-free PSE and the caffeinated C. liberica „dewevrei' (DEW), an oldcultivated species from the Central African Republic. This interspecific cross includes F1 hybrids and the two backcrosses F1 x PSE (BCPSE) and F1 x DEW (BCDEW). From BCPSE, a major gene with two alleles ( $C$ and $C$ ) has been found to control the presence or absence of caffeine in beans, with the absence being recessive (Barre et al. 1998). In heterozygote BCDEW hybrids (Cc), CAF is under polygenic control and these hybrids were consequently used in the study.

The quantitative trait locus (QTL) approach is efficient for highlighting major genes. In coffee trees, this approach generated the first evidence of the major gene Ft1, which concerns the fructification time (Akaffou et al. 2003). QTL evidence mainly depends on four parameters: 
the difference between parents for quantitative traits, the number of markers and descendants in the progeny, and the heritability. Although the number of markers can easily reach 200 and more nowadays, the difference between parents can only be maximized using interspecific crosses. This was the case for the fructification time, which represents another great difference between PSE and DEW (10 weeks in PSE vs 10 months in DEW). Unfortunately, when parental species are distant, the number of hybrids and their fertility are low. In some cases, the choice of an interspecific cross, where the parents differ markedly, can be more important than the progeny size, which is necessarily limited by the hybrid fertility. That is why the PSE-DEW cross is still very interesting material at the backcross level when studying CAF, a highly heritable trait, despite the relatively low hybrid number.

In this study, the QTL approach was combined with principal component analysis (PCA). The latter analysis allowed us to assess the number of independent components explaining CAF diversity within BCDEW. As each of these selected components was highly correlated with some traits, it was then possible to retain one trait per component showing the highest correlation, and then to look for its QTL. This approach revealed two independent QTLs explaining most of CAF diversity within BCDEW.

\section{Materials and Methods}

\subsection{Plant material}

The plant material (trees) was maintained at the IRD coffee research station (Man, Côted"Ivoire) $)^{1}$. The cross between C.pseudozanguebariae Bridson (PSE) and C.liberica dewevrei Hiern (DEW) was carried out using the PSE tree \# 8044, as female, handpollinated under bags using pollen from the DEW tree \# 5851. Twenty-five F1 hybrids were obtained and grown on the same plot at the station. Sixty-two backcross hybrids were obtained from $13 \mathrm{~F} 1$ hybrids (used as female parents) with DEW as pollen donor. For each $\mathrm{BCDEW}$ hybrids, its genetic belonging to BCDEW was checked using molecular markers (Ky et al. 2000).

\subsection{Biochemical and phenological data}

Biochemical data concerned caffeine (CAF) and chlorogenic acid content evaluations (sample preparation, extraction and HPLC dosage) and were recorded as described in Barre et al. (1998) and Ky et al. (1999), respectively. Chlorogenic acid content estimations concerned the following isomers : 5\&4-CQA ${ }^{2}, 3-C Q A, 3,5-d i C Q A, 3,4-d i C Q A, 4,5-d i C Q A, 5-$ FQA, 4-FQA and 3-FQA. These data led us to compute:

- the total CQA content, i.e. CQAt = 5\&4-CQA + 3-CQA;

- the total diCQA content, i.e. diCQAt = 3,5-diCQA + 3,4-diCQA + 4,5-diCQA;

- the total FQA content, i.e. FQAt $=5-F Q A+4-F Q A+3 F Q A$, but also:

- the total of caffeoyl and dicaffeoylquinic acids, i.e. CGA1 = CQAt + diCQAt,

- and the total of chlorogenic acids sensu largo, i.e. CGA2 = CGA1 + FQAt.

- By contrast, phenological data were evaluated at the Man coffee research station (Côted'Ivoire) and concerned the fructification growth period (FGP) and seed weight (SW) (Akaffou

\footnotetext{
${ }^{1} \ln 1997$

${ }^{2}$ Peaks of 5-CQA and 4-CQA overlapped, leading to the 5\&4-CQA estimation
} 
et al. 2003). Fructification growth period was the time, expressed in days, between the flowering and the end of the green bean growth.

Biochemical and phenological data also allowed us to compute CAF/CQAt, CAF/CGA1, CAF/CGA2, and CAF/SW. Finally, as Ft1 explains a part of CAF (Akaffou et al. 2003), a corrected CAF was estimated assuming the same FGP for each hybrid (215 days as in DEW): $C A F C=215 \times(C A F / F G P)$. Similarly, a corrected SW was also estimated $(S W C=215$ $x(S W / F G P)$

\subsection{Principal component analysis}

The principal component analysis (PCA; Varimax normalized) was applied to the biochemical and agronomical data table in order to detect a reduced independent number of factors (Hotelling 1933) that could explain the CAF variation within BCDEW. The Varimax normalized process involved two steps: $1 /$ estimating of the number $\mathrm{K}$ of components using the Kayser criteria (proper value > 1) from a classical CPA; and 2/ applying the Varimax normalized option assuming $\mathrm{K}$ components and leading to robust component estimation.

\subsection{Mapping and $Q T L$ identification}

The map construction was previously described (Ky et al. 2000). Single marker analysis (ANOVA) was first performed to detect QTLs with a probability level of $p<0.01$. The Statistica software package (version 6.1) was used for all of the statistical analyses, including PCA.

\section{Results}

\subsection{PCA analysis of the biochemical and phenological diversity within BCDEW hybrids}

The first objective was to analyze the biochemical and phenological diversity within BCDEW hybrids to highlight some independent factors linked to CAF. The data was first analyzed by classical PCA. Six components showed a proper value of over 1 (Kayser criteria) and they explained $92.6 \%$ of the generalized variance. Table 1 shows correlation coefficients between components and initial variables, whereas table 2 gives the $R^{2}$ values. The following interpretations are based on the data presented in both tables. Only the 4 first components, concerning chlorogenic acid and caffeine contents diversity, were presented in tables 1 and 2.

In addition, only two components, i.e. the first and fourth, were of interest as they explained $97.7 \%$ of the CAF variance (Tables $1 \& 2$ ). The first component was the most important in terms of $\mathrm{R}^{2}$, explaining $79 \%$ of the CAF variance (Table 2 ). The fourth component was highly correlated with the total CQA content (CQAt), explaining about $18.7 \%$ of the CAF variance. Note that the fruit growth period (FGP), i.e. Ft1, had little effect on CAF and only through the first component. From the analysis, two traits - CQAt and the ratio CAF/CQAt - were selected to explain the CAF variation.

\subsection{Relationships between CAF, CAF/CQAt and CQAt in BCDEW hybrids}

Figure 1 confirmed the absence of relationship between CQAt and the CAF/CQA ratio on one hand, and the relationships between CAF and CQAt, and between CAF and CAF/CQAt on the other. We then looked for QTLs for CAF/CQA and CQAt. 


\subsection{Quantitative analysis of the CAF/CQAt ratio and CQAt in parental species, F1, and backcross hybrids.}

Highly significant differences were recorded between PSE, BCPSE, F1, BCDEW and DEW groups for CAF/CQAt and the calculated averages were $0.0,0.0033,0.051,0.121$, and 0.155 units for PSE, BCPSE, F1, BCDEW, and DEW, respectively. The regression between the proportion of DEW genome in each of the five groups $(0 \%, 25 \%, 50 \%, 75 \%$, and $100 \%$, in PSE, BCPSE, F1, BCDEW, and DEW, respectively) and CAF/CQAt was linear when PSE was excluded from the analysis (Fig. 2). In fact, additivity prevailed when the $c$ allele (absence of caffeine) was not present in the homozygous state. By contrast with CAF/CQAt, additivity has been documented for CQAt and applies even when the $c$ allele is present in the homozygous state (Ky et al. 1999).

The next objective was to describe the phenotypic distribution of CAF/CQAt within the BCDEW progeny. The ratio ranged from 0.050 to 0.214 ( 0.121 on average). Especially important, its distribution (Fig. 3A) seemed bimodal, suggesting a major gene. CQAt ranged from 3.18 to $6.25 \% \mathrm{dmb}(4.41 \% \mathrm{dmb})$ in $\mathrm{BCDEW}$ and its distribution was unimodal (Fig. 3B).

\subsection{Identification of QTLs for CAF/CQAt and CQAt}

One QTL, i.e. RCQ1 $\left(F_{1,43}=17.5 ; p=0.00014\right)$, was detected for the ratio It was located on linkage group A, $7.2 \mathrm{cM}$ from the AACCAC10 marker and $17.2 \mathrm{cM}$ from the AACCTT4 marker (Fig. 4 A). Segregation of the AACCAC10 marker was 15:17, indicating Mendelian segregation without distortion.

Evidence of another QTL, named CQA1, was obtained on linkage group $G(F 1,51=12.0 ; p$ $=0.0011$ ), $0.8 \mathrm{cM}$ from the ACTCAT5 marker and $0.8 \mathrm{cM}$ from the ACACTT1 marker (Fig. 4 B). In particular, there was no interaction between QTLs, thus confirming their genetic and physiological independence. Finally, no segregation distortion was observed for AACCAC10 (21 vs 24) and ACTCAT5 (17 vs 26). [D1D1] and [D1P1] represent the two BCDEW genotypes at the ACTCAT5 locus and [D2D2] and [D2P2] are the two BCDEW genotypes at the AACCAC10 locus. CAFs were then estimated for genotypes [D1D1 D2D2], [D1D1 D2P2], [D1P1 D2D2] and [D1P1 D2P2], i.e. 0.74, 0.53, 0.58, and 0.39, respectively.

\section{Discussion}

The main finding was the evidence of two independent processes responsible for most of the CAF variation within BCDEW. Importantly, each process could be characterized by two highly independent correlated traits, i.e. CAF/CQAt and CQAt, for which the corresponding QTL was detected on two independent linkage groups.

\subsection{New hypotheses explaining caffeine content variation}

In the Coffea genus, two main groups of species can be distinguished according to the presence or not of caffeine in their green seeds. "Caffeine-free" species represent about half of the Coffea genus and are present throughout the distribution area of wild coffee plants, although its frequency is higher in Madagascar (Charrier and Berthaud, 1975). In fact, the term "caffeine-free" is not exact since traces of caffeine may be found in their seeds $(<0.01 \%$ dmb) (Campa et al. 2005a). Very low CAF varieties also exist, probably as natural mutants, in Ethiopian C. arabica, and this is due to the presence of an inactive caffeine synthase (Sivarolla et al. 2004).

An interspecific cross between a caffeine-free species (PSE) and a caffeinated species (DEW) has revealed a major gene governing the presence or absence of caffeine, with the 
absence being due to the presence of the recessive $c$ allele in the homozygous state (Barre et al. 1998). However, this is not sufficient to explain the CAF variation between BCDEW hybrids (Barre et al. 1998) or between caffeinated species (Campa et al. 2005a). In the current investigation, two independent QTLs-RCQ1 and CQA1-were found to be responsible for the CAF variation in caffeinated seeds. In particular, they were located on two linkage groups, indicating genetic independence. It was then possible to define the components first and fourth as the functional impact of RCQ1 and CQA1. For each hybrid, the CAF detected in their seeds was the result of both RCQ1 and CQA1.

The relationship between CQAt and CAF in wild species is well known (Anthony et al. 1993), i.e. CAF increases as CQAt increases. Nevertheless, the relationship is not absolute and there are also marked variations in the CAF/CQAt ratio between wild species (Campa et al. 2005b). The similarity in the organization of CAF and CQAt diversity among species and within BCDEW was thus an important finding. At the molecular level, there is less caffeine than chlorogenic acid in Coffea seeds and only a part of the chlorogenic acid seems to be complexed (Campa et al. 2005b). Under this hypothesis, RCQ1 would regulate the ratio between CAF and CQAt.

Biological interpretation of the effects of CQAt and RCQ1 was not easy. Nevertheless, this problem was partly overcome by removing some putative candidate genes. For example, concerning CQA1, the two genes CCPAL1 and CCCOAOMT1 were not located on the linkage group G (Campa et al. 2003; Mahesh et al. 2006) and cannot be candidate for CQA1. By contrast, they were located on the linkage group $A$, but at more than $50 \mathrm{cM}$ from $R C Q 1$. Nonetheless, both genes CCPAL1 and CCCOAOMT1 explained part of the CQAt variation, suggesting a physiological effect downstream of CQA1.

Similarily, Ft1 the major gene explaining FGP, was not located on linkage group $\mathrm{G}$ or linkage group A. Its low impact on CAF (Akaffou et al. 2003) seems to be related to its low correlation between the first component and FGP (Table 1), but interpretation of this effect is untimely.

\subsection{Efficiency of the two-step analysis - PCA-QTL}

The first PCA analysis steps allowed us to determine the number of independent factors responsible for within-BCDEW variations for CAF. Once the number was estimated, it was possible to look for QTLs for some traits representative of each component. This procedure has permit to reach easily objectives.

The marked difference between parents and the high heritability of CAF (Barre et al. 1998) were sufficient to highlight two independent QTLs, despite the relatively low number of hybrids. Certainly the accuracy concerning the location could be relatively low, but this was not assessed in the present study.

\section{References}

Akaffou S, Ky CL, Barre P, Hamon S, Louarn J, Noirot M (2003) Identification and mapping of a major gene (Ft1) involved in fructification time in the interspecific cross Coffea pseudozanguebariae x C. liberica var. Dewevrei: impact on caffeine content and seed weight. Theor Appl Genet 106: 1486-1490

Anthony F, Clifford MN, Noirot M (1993) Biochemical diversity in the genus Coffea L.: chlorogenic acids, caffeine, and mozambioside contents. Genet Res Crop Evol 40: 61-70 
Barre P, Akaffou S, Louarn J, Charrier A, Hamon S, Noirot M (1998) Inheritance of caffeine and heteroside contents in an interspecific cross between a cultivated coffee species Coffea liberica var dewevrei and a wild species caffeine-free C. pseudozanguebariae. Theor Appl Genet 96:306-311

Campa C, Noirot M, Bourgeois M, Pervent M, Ky C-L, Chrestin H, Hamon S, de Kochko A (2003) Genetic mapping of a caffeoyl-coenzyme A 3-O-methyltransferase gene in coffee trees. Impact on chlorogenic acid content. Theor Appl Genet 107:751-756

Campa C, Doulbeau S, Dussert S, Hamon S, Noirot M (2005a) Diversity in bean caffeine content among wild Coffea species: evidence of a discontinuous distribution. Food Chem 91:633-637

Campa C, Doulbeau S, Dussert S, Hamon S, Noirot M (2005b) Qualitative relationship between caffeine and chlorogenic acid contents among wild Coffea species. Food Chem, 93:135-139

Charrier A, Berthaud J (1975) Variation de la teneur en caféine dans le genre Coffea. Café Cacao Thé 19:251-264

Hotteling H (1933) Analysis of a complex of statistical variables into principal components. J Educ Psychol 24:417 441

Ky CL, Louarn J, Guyot B, Charrier A, Hamon S, Noirot M (1999) Relations between and inheritance of chlorogenic acid content in an interspecific cross between Coffea pseudozanguebariae and Coffea liberica var „dewevrei". Theor Appl Genet 98:628-637

Ky CL, Barre P, Lorieux M, Trouslot P, Akaffou S, Louarn J, Charrier A, Hamon S, Noirot M (2000) Interspecific genetic linkage map, segregation distortion and genetic conversion in coffee (Coffea sp.). Theor Appl Genet 101:669-676

Ky CL, Louarn J, Dussert S, Guyot B, Hamon S, Noirot M (2001) Caffeine, trigonelline, chlorogenic acids and sucrose diversity in wild Coffea arabica L. and C. canephora P. accessions. Food Chem 75: 223-230

Mahesh V, Rakotomalala JJ, Le Gal L, Vigne H, de Kochko A, Hamon S, Noirot M, Campa C (2006) Isolation and genetic mapping of a Coffea canephora phenylalanine ammonia-lyase gene (CCPAL1) and its involvement in the accumulation of caffeoyl quinic acids. Plant Cell Rep 25:986-992

Mazzafera P, Sivarolla MB, Alves de Lima MM, Medina Filho P (1997) Caffeine content of diploid coffee species. Cienca et cultura 49:216-218

Mizuno K, Okuda A, Kato M, Yoneyama N, Tanaka H, Ashihara H, Fujimura T (2003) Isolation of a new dual-functional caffeine synthase gene encoding an enzyme for the conversion of 7-methylxanthine to caffeine from coffee (Coffea arabica L.). FEBS Lett $534: 75-81$

Montagnon C, Guyot B, Cilas C, Leroy T (1998) Genetic parameters of several biochemical compounds from green coffee, Coffea canephora. Plant Breed 117:576-578

Nehlig A, Daval JL, Debry G (1992) Caffeine and the central nervous system: mechanisms of action, biochemical, metabolic and psychostimulant effects. Brain Res Rev 17:139-170

Nehlig A (2012) Effets physiologiques du café et santé humaine. Une revue. Cah Agric 21:107- 2007 
Ogawa M, Herai Y, Koizumi N, Kusano T, Sano H (2001) 7-methylxanthine methyltransferase of coffee plants. Gene isolation and enzymatic properties. $\mathrm{J}$ biol Chem 276: 8213-8218

Ogita S, Uefuji H, Yamaguchi Y, Koizumi N, Sano H (2003) Producing decaffeinated coffee plants. Nature 423:823

Payen A (1846) Premier mémoire sur le café. C-R Acad Sci, Paris 22:724-737

Sivarolla MB, Mazzafera P, Fazuoli LC (2004) A naturally decaffeinated arabica coffee. Nature 429:826

Sondheimer E, Covitz F, Marquisee MJ (1961) Association of naturally occuring compounds, the chlorogenic acid-caffeine complex. Arch Biochem Biophys 93:63-71

Uefuji H, Ogita S, Yamaguchi Y, Koizumi N, Sano H (2003) Molecular cloning and functional characterization of three distinct $\mathrm{N}$-methyltransferases involved in the caffeine biosynthetic pathway in coffee plants. Plant Physiol 132: 372-380 


\section{Figures}

Figure 1. Relationships between A) CAF/CQAt and CQAt, B) CAF and CAF/CQAt, and C) CAF and CQAt
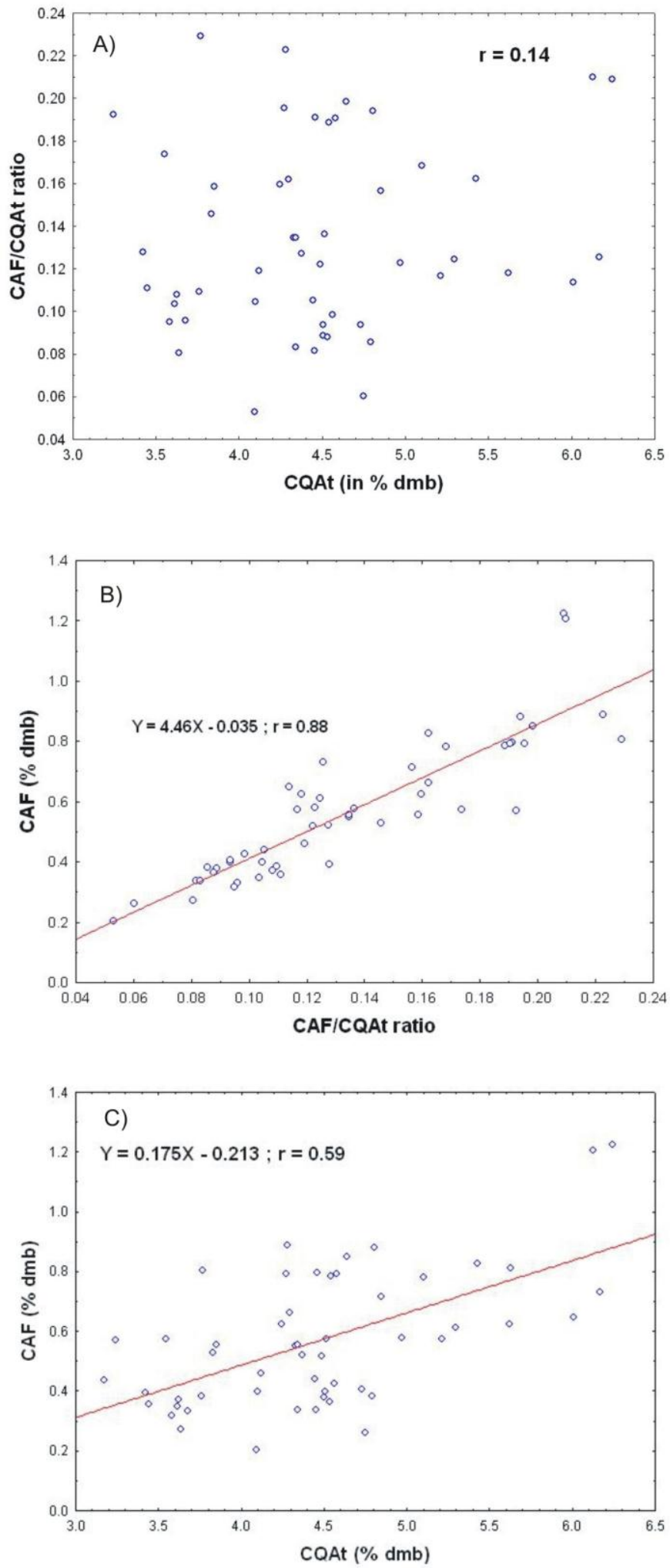
Figure 2. Additivity in the CAF/CQAt ratio

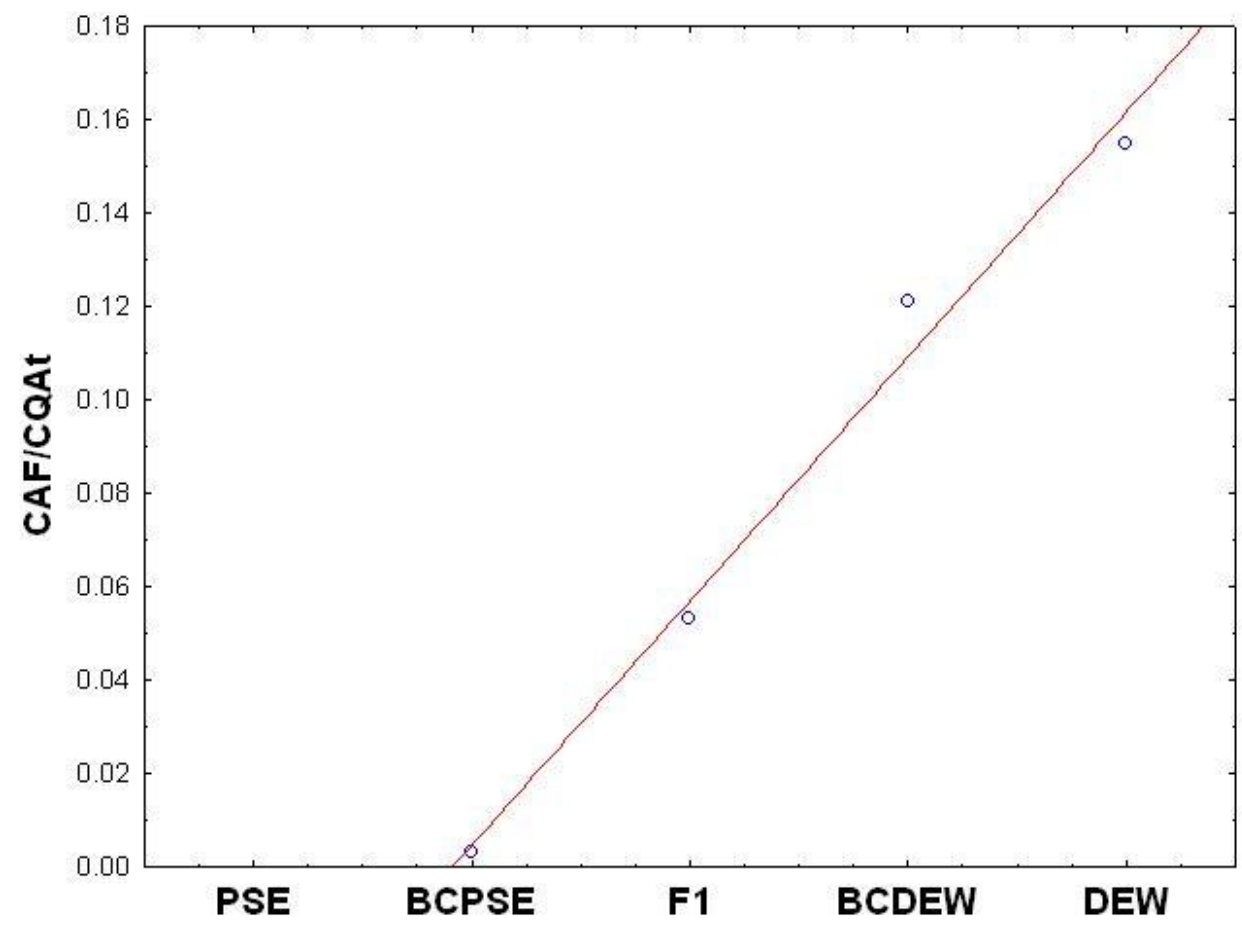


Figure 3. Distribution of $(A)$ the CAF/CQA and (B) CQAt ratios within the BCDEW progeny.
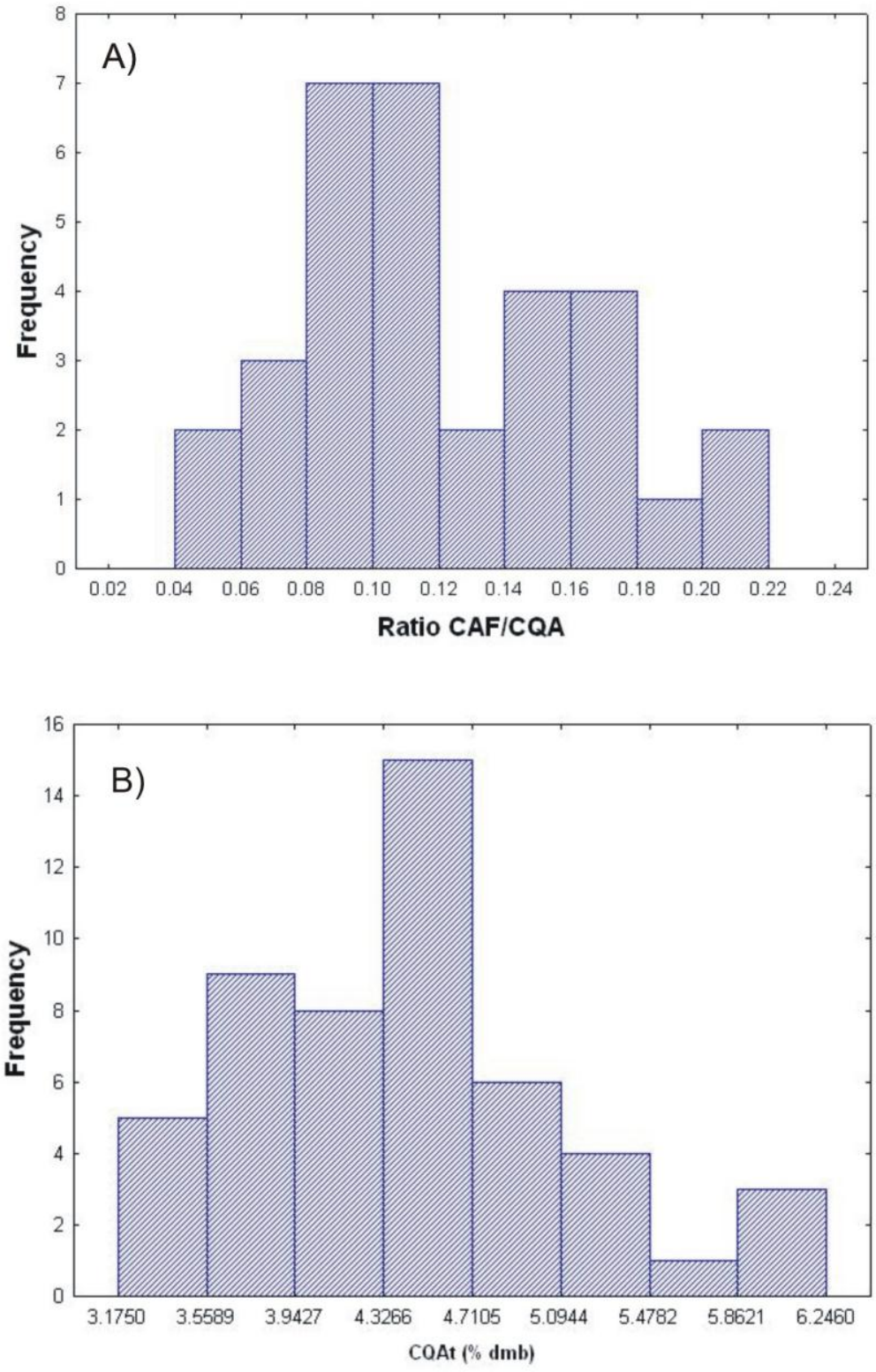
Figure 4. Location of the QTLs RCQ1 and CQA1 on linkage groups $A$ and $\mathrm{G}$, respectively (genetic map from Ky et al. 2000). Only a part of the linkage group A is depicted here. Distances (in $\mathrm{cM}$ ) are given on the left of each linkage group, while marker names are on the right.
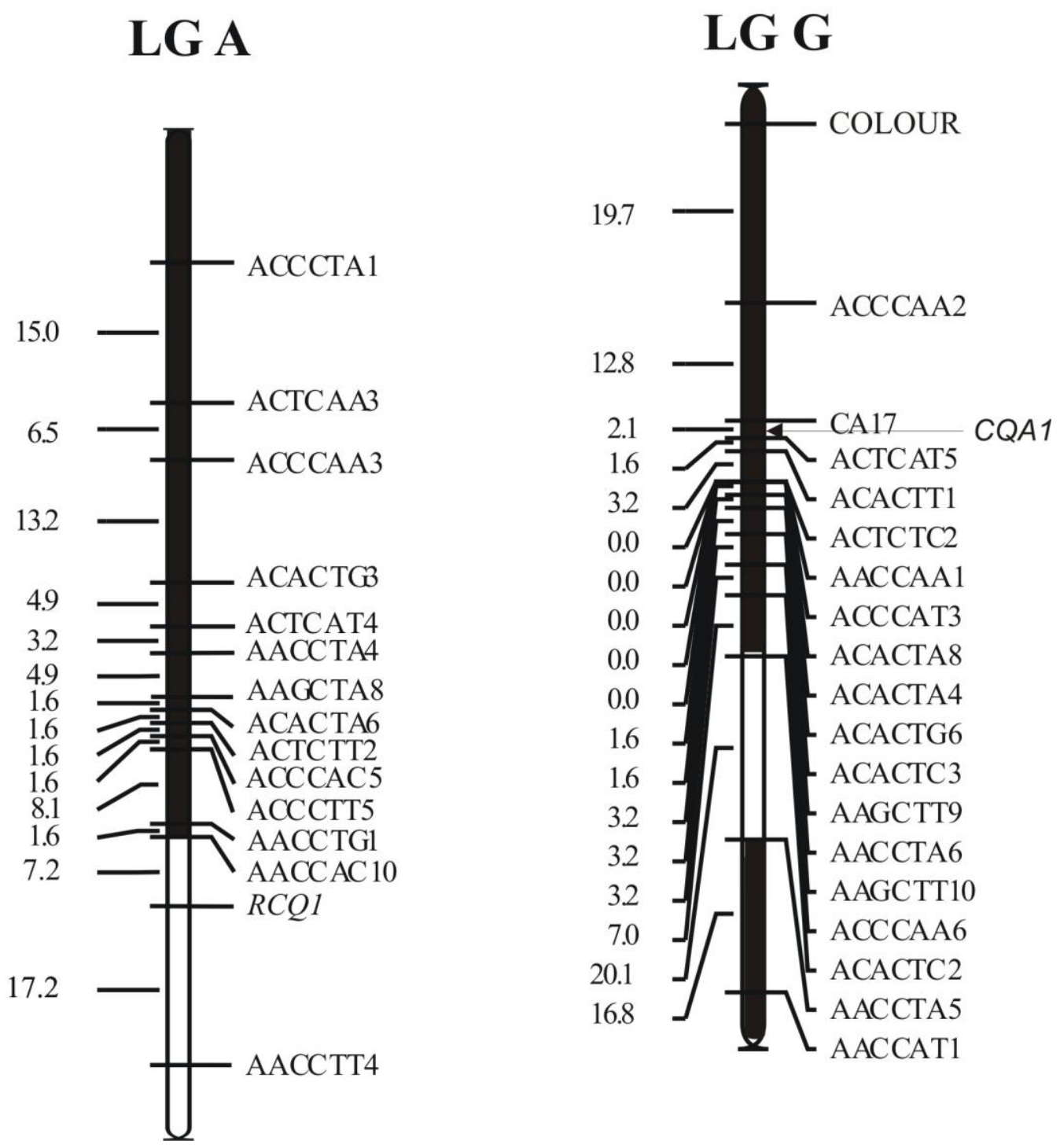
Table 1. Correlations between principal components and initial variables. When absolute values are higher than 0.70 , the correlations are in bold

\begin{tabular}{|l|c|c|c|c|}
\hline & PCA 1 & PCA 2 & PCA 3 & PCA 4 \\
\hline FGP & 0.338 & 0.033 & -0.098 & -0.087 \\
\hline SW & 0.144 & -0.215 & 0.093 & 0.051 \\
\hline SWC & 0.070 & -0.280 & 0.281 & 0.059 \\
\hline 3-CQA & 0.097 & -0.187 & -0.175 & 0.599 \\
\hline 4\&5-CQA & 0.182 & 0.074 & -0.059 & $\mathbf{0 . 9 6 0}$ \\
\hline CQAt & 0.183 & 0.055 & -0.072 & $\mathbf{0 . 9 6 9}$ \\
\hline 3-FQA & 0.068 & -0.112 & $\mathbf{0 . 8 9 8}$ & -0.172 \\
\hline 4-FQA & -0.051 & 0.058 & $\mathbf{0 . 7 8 5}$ & 0.119 \\
\hline 5-FQA & 0.079 & -0.065 & $\mathbf{0 . 9 2 9}$ & -0.059 \\
\hline FQAt & 0.060 & -0.050 & $\mathbf{0 . 9 7 2}$ & -0.038 \\
\hline 3,5-diCQA & 0.087 & 0.593 & -0.371 & 0.391 \\
\hline 3,4-diCQA & -0.033 & $\mathbf{0 . 9 3 0}$ & 0.034 & -0.051 \\
\hline 4,5-diCQA & -0.001 & $\mathbf{0 . 9 3 8}$ & 0.001 & 0.209 \\
\hline DiCQAt & 0.010 & $\mathbf{0 . 9 6 5}$ & -0.081 & 0.202 \\
\hline CGA1 & 0.166 & 0.264 & -0.082 & $\mathbf{0 . 9 3 9}$ \\
\hline CGA2 & 0.184 & 0.252 & 0.161 & 0.929 \\
\hline CAF & $\mathbf{0 . 8 8 9}$ & 0.045 & 0.033 & 0.433 \\
\hline CAF/CQAt & $\mathbf{0 . 9 6 8}$ & -0.008 & 0.097 & -0.023 \\
\hline CAF/CGA1 & $\mathbf{0 . 9 6 8}$ & -0.076 & 0.105 & 0.037 \\
\hline CAF/CGA2 & $\mathbf{0 . 9 6 9}$ & -0.066 & -0.024 & 0.084 \\
\hline CAFC & $\mathbf{0 . 8 3 6}$ & 0.058 & -0.025 & 0.407 \\
\hline CAF/SW & $\mathbf{0 . 8 2 5}$ & 0.161 & -0.038 & 0.414 \\
\hline
\end{tabular}


Table 2. Relative contributions $\left(\mathrm{R}^{2}\right)$ between principal components and initial variables. When absolute values are higher than 0.30 , contributions are in bold

\begin{tabular}{|l|c|r|r|r|}
\hline & \multicolumn{1}{|l|}{ PCA 1 } & \multicolumn{1}{l|}{ PCA 2 } & PCA 3 & PCA 4 \\
\hline FGP & 0.114 & 0.001 & 0.010 & 0.008 \\
\hline SW & 0.021 & 0.046 & 0.009 & 0.003 \\
\hline SWC & 0.005 & 0.078 & 0.079 & 0.003 \\
\hline 3-CQA & 0.009 & 0.035 & 0.031 & $\mathbf{0 . 3 5 9}$ \\
\hline 4\&5-CQA & 0.033 & 0.005 & 0.003 & $\mathbf{0 . 9 2 2}$ \\
\hline CQAt & 0.033 & 0.003 & 0.005 & $\mathbf{0 . 9 3 9}$ \\
\hline 3-FQA & 0.005 & 0.013 & $\mathbf{0 . 8 0 6}$ & 0.030 \\
\hline 4-FQA & 0.003 & 0.003 & $\mathbf{0 . 6 1 6}$ & 0.014 \\
\hline 5-FQA & 0.006 & 0.004 & $\mathbf{0 . 8 6 3}$ & 0.003 \\
\hline FQAt & 0.004 & 0.003 & $\mathbf{0 . 9 4 5}$ & 0.001 \\
\hline 3,5-diCQA & 0.008 & $\mathbf{0 . 3 5 2}$ & 0.138 & 0.153 \\
\hline 3,4-diCQA & 0.001 & $\mathbf{0 . 8 6 5}$ & 0.001 & 0.003 \\
\hline 4,5-diCQA & 0.000 & $\mathbf{0 . 8 8 0}$ & 0.000 & 0.044 \\
\hline DiCQAt & 0.000 & $\mathbf{0 . 9 3 1}$ & 0.007 & 0.041 \\
\hline CGA1 & 0.028 & 0.070 & 0.007 & $\mathbf{0 . 8 8 2}$ \\
\hline CGA2 & 0.034 & 0.064 & 0.026 & $\mathbf{0 . 8 6 3}$ \\
\hline CAF & $\mathbf{0 . 7 9 0}$ & 0.002 & 0.001 & 0.187 \\
\hline CAF/CQAt & $\mathbf{0 . 9 3 7}$ & 0.000 & 0.009 & 0.001 \\
\hline CAF/CGA1 & $\mathbf{0 . 9 3 7}$ & 0.006 & 0.011 & 0.001 \\
\hline CAF/CGA2 & $\mathbf{0 . 9 3 9}$ & 0.004 & 0.001 & 0.007 \\
\hline CAFC & $\mathbf{0 . 6 9 9}$ & 0.003 & 0.001 & 0.166 \\
\hline CAF/SW & $\mathbf{0 . 6 8 1}$ & 0.026 & 0.001 & 0.171 \\
\hline
\end{tabular}

